\title{
A test for radial mixing using local star samples
}

\author{
Jincheng Yu ${ }^{1}$, Jerry Sellwood ${ }^{2}$, Carlton Pryor ${ }^{2}$, Li Chen ${ }^{1}$ and \\ Jinliang $\mathbf{H o u}^{1}$ \\ ${ }^{1}$ Shanghai Astronomical Observatory, Chinese Academy of Sciences, China, \\ email: yujc@shao.ac.cn \\ ${ }^{2}$ Department of Physics and Astronomy, Rutgers University
}

\begin{abstract}
We use samples of local main-sequence stars to show that the radial gradient of $[\mathrm{Fe} / \mathrm{H}]$ in the thin disk of the Milky Way decreases with mean effective stellar temperature. We use the angular momentum of each star about the Galactic center to eliminate the effects of epicyclic motion, which would otherwise blur the estimated gradients. We use the effective temperatures as a proxy for mean age, and conclude that the decreasing gradient is consistent with the predictions of radial mixing due to transient spiral patterns. We find some evidence that the trend of decreasing gradient with increasing mean age breaks to a constant gradient for samples of stars whose main-sequence life-times exceed the likely age of the thin disk.
\end{abstract}

